\title{
Alterations of glomerular basement membrane charge and structure in diabetic nephropathy
}

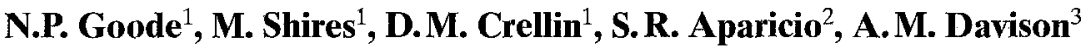 \\ ${ }^{1}$ Renal Research Unit, St. James's University National Health Service Trust, Leeds, UK \\ ${ }^{2}$ Department of Pathology, St. James's University National Health Service Trust, Leeds, UK \\ ${ }^{3}$ Department of Renal Medicine, St. James's University National Health Service Trust, Leeds, UK
}

\begin{abstract}
Summary We examined glomerular basement membrane anionic site distribution identified by cationic gold in seven patients with insulin-dependent and four patients with non-insulin-dependent diabetes mellitus, presenting a spectrum of clinical and glomerular changes. Anionic sites were investigated by pretreatment of tissue with glycosaminoglycan-degrading enzymes prior to cationic gold staining. The distribution of chondroitin sulphate proteoglycans a previously unrecognized glomerular basement membrane component - and type IV collagen was examined by immunoelectron microscopy to identify structural changes in the basement membrane. Findings were compared with those of non-diabetic patients showing minor proteinuria and morphologically normal glomerular basement membranes. Two patients, originally diagnosed as having diabetic nephropathy were also examined at 19 weeks and 5 years after renal transplantation. Characteristic redistribution of type IV collagen and chondroitin sulphate proteoglycans was noted in thickened glomerular basement membrane segments $(>400 \mathrm{~nm})$ of diabetic patients and those with renal transplants. Exten-
\end{abstract}

sion of anionic sites deep into the glomerular basement membrane at $\mathrm{pH} 2.5$, together with loss of interna sites at $\mathrm{pH} 5.8$ is unique to diabetic nephropathy. Reduced charge density was apparent in some patients due to thickening of the glomerular basement membrane, although the number of anionic sites per unit length of membrane was actually increased. Thus, charge aberration in diabetic nephropathy is due to displacement rather than loss of anionic sites. Removal of more than $90 \%$ of these sites by heparitinase, confirms their association with heparan sulphate proteoglycans. Similar derangement of anionic sites in all patients with diabetic nephropathy irrespective of the degree of proteinuria, suggests that a heparan sulphate proteoglycan-related charge barrier plays a minor role in controlling permeability of the diabetic glomerular basement membrane. [Diabetologia (1995) 38: 1455-1465]

Key words Anionic site, cationic gold, heparan sulphate proteoglycans, chondroitin sulphate proteoglycans, collagen IV.
Received: 10 November 1995 and in final revised form: 14 June 1995

Corresponding author: Dr. N.P. Goode, Renal Research Unit, Clinical Sciences Building, St. James's University N.H.S. Trust, Leeds LS9 7TF, UK

Abbreviations: BSA, Bovine serum albumin; CG, cationic gold; CSPG, chondroitin sulphate proteoglycans; GAG, glycosaminoglycan; GBM, glomerular basement membrane; HSPG, heparan sulphate proteoglycans; LRE, lamina rara externa; LRI, lamina rara interna; PCI, protein:creatinine index.
A major complication of diabetes mellitus is nephropathy leading to the nephrotic syndrome and ultimately to renal failure. Renal biopsies from patients who have progressed to overt clinical nephropathy histologically appear to range from minor changes to diffuse or nodular glomerulosclerosis. Characteristic morphological features of diabetic nephropathy are seen in non-occluded glomerular capillary loops as a thickening of the glomerular basement membrane (GBM) and expansion of mesangial matrix [1].

Alterations in the biochemical composition of the GBM in diabetes, (reviewed previously [2]), include 
accumulation of type IV collagen [3-5] and other non-enzymatically glycated proteins [6], reduced synthesis of sulphated glycosaminoglycans $[7,8]$, and a reduced content of sialic acid [9], laminin [10] and heparan sulphate proteoglycan (HSPG) $[5,11,12]$. These changes in macromolecular composition of the GBM are assumed to be the basis for a loss of size and charge selectivity, shown by functional studies to occur in diabetes associated with proteinuria [13].

Glomerular mesangial, endothelial and visceral epithelial cells are believed to contribute to GBM biogenesis and degradation $[14,15]$. In diabetic nephropathy, GBM thickening and mesangial matrix expansion may be due to either increased synthesis or decreased degradation of intrinsic components, accumulation of extrinsic components, or reduced component density. This altered molecular architecture of the diabetic GBM may be due to a differential response of glomerular cells to conditions imposed by the diabetic milieu.

We have previously reported the influence of different glomerulopathies on the distribution of cationic colloidal gold (CG) stained anionic sites in post-embedded renal biopsy tissue [16]. In the present study, we have used this technique in conjunction with immunogold staining of chondroitin sulphate proteoglycans - a mesangial matrix component previously believed not to be a constituent of normal mature GBM [7, 17-19], and type IV collagen - the major collagenous component of the GBM, to investigate the influence of macromolecular restructuring on the distribution of anionic sites in the diabetic GBM. The nature of these sites was further examined by the influence of glycosaminoglycan (GAG) degrading enzymes on charge staining patterns.

\section{Subjects, materials and methods}

Tissue. Renal biopsy tissue $\left(1 \mathrm{~mm}^{3}\right)$ from seven patients with insulin-dependent and four patients with non-insulin-dependent diabetes mellitus, two diabetic patients following renal transplantation, and three non-diabetic patients with minor protein excretion (protein:creatinine index $[\mathrm{PCI}]<1000$ ) but showing no glomerular pathological changes and normal GBM morphology (Table 1), was processed into LR Gold ${ }^{\circledR}$ resin (London Resin Company, Basingstoke, Hampshire, U. K.) as previously described [16]. In the absence of normal biopsy tissue, GBM from this latter group will be referred to as "normal" for descriptive purposes.

PCI was calculated as urinary protein concentration $(\mathrm{g} / \mathrm{l}) \times 1000$, divided by the urinary creatinine concentration $(\mathrm{mmol} / 1)[20]$.

Cationic gold staining. The CG marker was prepared from a bovine serum albumin (BSA)-stabilized poly-L-lysine-colloidal gold complex as previously described [21]. Ultrathin tissue sections, mounted on formvar-coated $300 \mu \mathrm{m}$ single hole gold grids were stained with cationic colloidal at $\mathrm{pH} 2.5$ as described previously [16], or at $\mathrm{pH} 5.8$ in a $0.02 \mathrm{~mol} / \mathrm{l}$ acetate buffer con-
Table 1. Clinical and demographic data in diabetic, renal transplant and "normal" patients

\begin{tabular}{llccc}
\hline Patient & $\begin{array}{l}\text { Age/sex } \\
\text { (male, female) }\end{array}$ & $\begin{array}{l}\text { Diabetic } \\
\text { history } \\
\text { (years) }^{\mathrm{b}}\end{array}$ & $\begin{array}{l}\text { Protein/ } \\
\text { creatinine } \\
\text { index }\end{array}$ & $\begin{array}{l}\text { Creatinine } \\
(\mu \mathrm{mol} / \mathrm{l})\end{array}$ \\
\hline $\begin{array}{l}\text { Minor diffuse changes } \\
1\end{array}$ & $59 / \mathrm{M}^{\mathrm{a}}$ & $1 / 52^{\mathrm{c}}$ & 378 & 504 \\
2 & $29 / \mathrm{M}$ & 7 & 366 & 248 \\
3 & $51 / \mathrm{F}$ & 38 & 1000 & 147 \\
4 & $64 / \mathrm{F}$ & 20 & 5812 & 220 \\
5 & $60 / \mathrm{M}^{\mathrm{a}}$ & 15 & 1000 & 601 \\
Diffuse & or nodular sclerosis & & & \\
6 & $60 / \mathrm{M}$ & 22 & 10163 & 667 \\
7 & $53 / \mathrm{M}$ & 3 & 8333 & 863 \\
8 & $54 / \mathrm{M}^{\mathrm{a}}$ & 3 & 18492 & 200 \\
9 & $64 / \mathrm{F}$ & 1 & 1788 & 103 \\
10 & $62 / \mathrm{F}$ & 15 & 8929 & 185 \\
11 & $49 / \mathrm{F}^{\mathrm{a}}$ & 20 & 20455 & 58 \\
Renal transplant & & & \\
12 & $42 / \mathrm{M}$ & $19 / 52^{\mathrm{d}}$ & 540 & 187 \\
13 & $54 / \mathrm{M}$ & $5^{\mathrm{d}}$ & 5231 & 160 \\
"Normal" GBM morphology & & \\
14 & $18 / \mathrm{M}$ & - & 117 & 90 \\
15 & $32 / \mathrm{F}$ & - & 140 & 72 \\
16 & $17 / \mathrm{F}$ & - & 815 & 120 \\
\hline
\end{tabular}

${ }^{\mathrm{a}}$ Non-insulin-dependent diabetes;

${ }^{b}$ history in years unless otherwise stated $(n / 52=$ no. of weeks $)$; ${ }^{c}$ diagnosed on admission;

${ }^{\mathrm{d}}$ post-transplant interval

taining $0.2 \mathrm{~mol} / \mathrm{l}$ magnesium chloride. In preliminary studies, this buffer system was shown to produce a similar distribution of CG-stained sites in the GBM to that reported previously using a Tris buffer, $\mathrm{pH}$ 7.0, with no additional electrolyte [16]. However, the modified buffer system reduced cytoplasmic and nuclear staining and resulted in a cleaner, more distinctive GBM charge pattern. The buffer conditions are similar to the critical electrolyte concentration for the demonstration of sulphated proteoglycans, first described by Scott and Dorling [22].

Enzyme digestion. GAG degrading enzymes, heparitinase I (EC 4.2.2.8) and chondroitinase AC from Flavobacterium heparinum (EC 4.2.2.5), were purchased from Sigma UK Limited (Poole, Dorset, UK).

Heparitinase I. Ultrathin tissue sections were incubated for $2 \mathrm{~h}$ at $43^{\circ} \mathrm{C}$ with 10 units $/ \mathrm{ml}$ heparitinase dissolved in $1.0 \mathrm{~N}$ sodium acetate buffer, $\mathrm{pH} 7.5$, containing $10 \mathrm{mmol} / \mathrm{l}$ calcium acetate and protease inhibitors $5 \mathrm{mmol} / \mathrm{l}$ benzamidine $\mathrm{HCl}$ and 0.1 mol/l 6-amino-n caproic acid [12] (Sigma UK).

Chondroitinase $A C$. Sequential sections were incubated for $90 \mathrm{~min}$ at $37^{\circ} \mathrm{C}$ with 0.5 units $/ \mathrm{ml}$ chondroitinase $\mathrm{AC}$ dissolved in $0.05 \mathrm{~mol} / 1$ Tris buffered saline, $\mathrm{pH} 7.3$, containing $0.04 \mathrm{~mol} / \mathrm{l}$ calcium chloride, $0.05 \% \mathrm{BSA}$ and the same protease inhibitors as listed for the heparitinase enzyme [12]. Enzyme digestion control sections were incubated in the absence of enzyme in either the heparitinase or the chondroitinase buffer system. All sections were washed in distilled water before staining in $\mathrm{CG}$ at $\mathrm{pH} 2.5$ or 5.8 , according to the routine procedure alongside untreated sections for comparison [16].

In dot-blot assays used for the examination of antibody specificity, native chondroitin sulphate A (Sigma UK) was digested for $90 \mathrm{~min}$ at $37^{\circ} \mathrm{C}$ with 0.5 units $/ \mathrm{ml}$ chondroitinase AC dissolved in the same buffer system (described above) used for tissue digestion. 


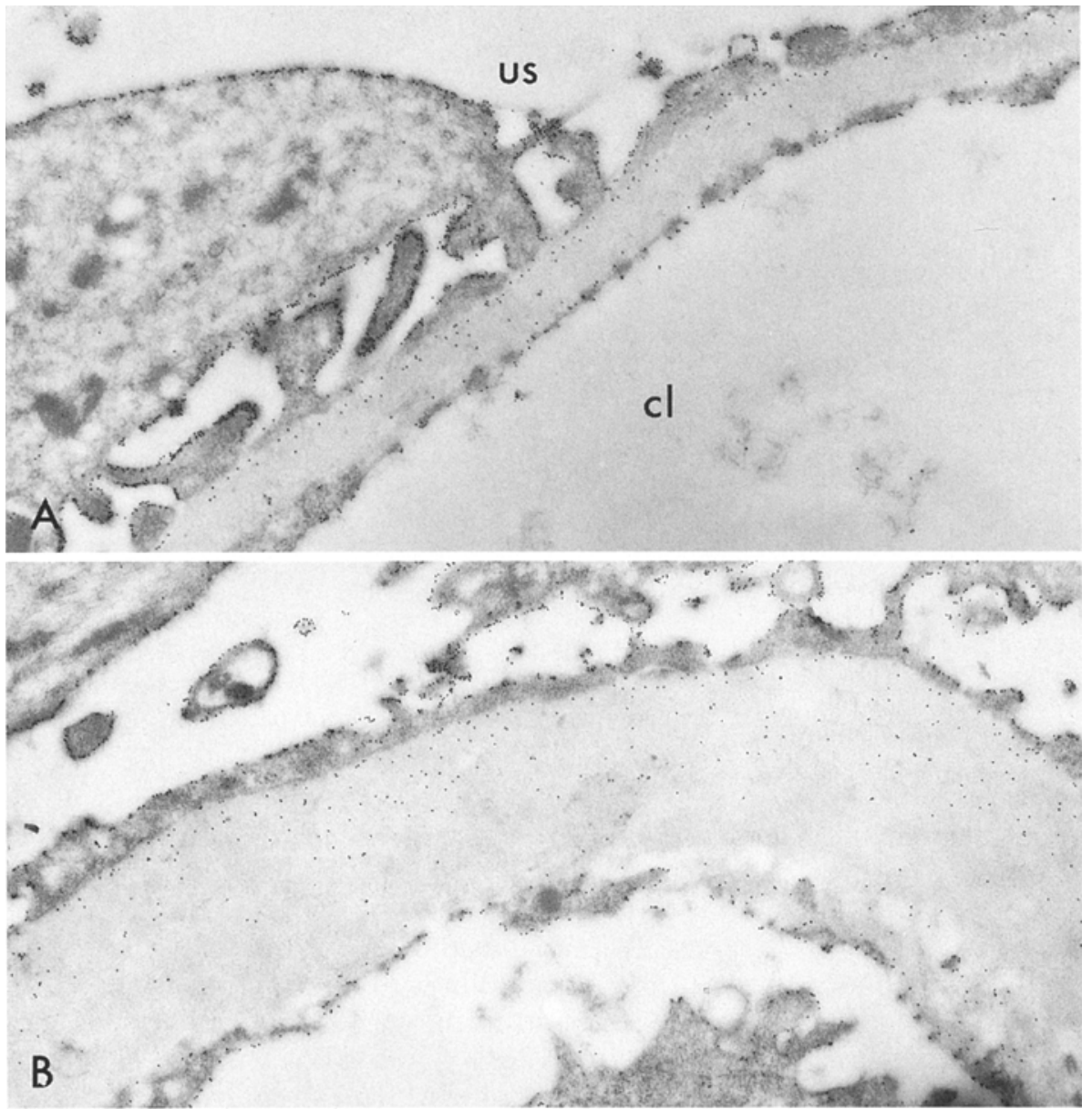

Fig. 1A, B. Anionic sites demonstrated by $\mathrm{CG}$ at $\mathrm{pH} 2.5$. A A linear array of externa sites is seen in "normal" GBM $(34,250 \times)$. B In diabetic GBM, anionic sites are present in a random pattern which extends into the deeper layers of the thickened membrane, patient $4(31,500 \times)$. cl, Capillary lumen; us, urinary space

\section{Immunogold staining}

Chondroitin sulphate proteoglycans. Ultrathin sections were stained by an indirect immunogold technique using a rabbit anti-chondroitin sulphate proteoglycans (CSPG) antiserum (Chemicon International Inc., Harrow, Middlesex, U.K.). Since native CSPG is poorly immunogenic [23], the antibody was raised against the highly antigenic GAG "stub" left on the core protein after chondroitinase digestion [24]. This antibody has been reported to have no reactivity against either the core protein, or the GAG side chains alone [24]. However, in this laboratory the antiserum was shown to bind to chondroitin sulphate A chains in a dot-blot assay, using a nitrocellulose membrane. Specificity was confirmed against doubling dilutions of a $1 \mathrm{mg} / \mathrm{ml}$ solution of native and enzyme-digested chondroitin sulphate A. Substrate-bound antibody was localized using goat anti-rabbit IgG coupled to $10 \mathrm{~nm}$ colloidal gold which was enhanced using silver lactate as developer [25]. The detection limit of a 1:500 dilution of antibody was $250 \mu \mathrm{g} / \mathrm{ml}$ of native chondroitin sulphate A, compared with $62.5 \mu \mathrm{g} / \mathrm{ml}$ of digested substrate.

Tissue sections were preincubated with 0.5 units $/ \mathrm{ml}$ of the enzyme in $0.05 \mathrm{~mol} / \mathrm{l}$ Tris buffered saline, $\mathrm{pH} 7.3$, containing $0.04 \mathrm{~mol} / \mathrm{l}$ calcium chloride for $1.5 \mathrm{~h}$ at $37^{\circ} \mathrm{C}$, jet-washed with distilled water and incubated overnight at $4^{\circ} \mathrm{C}$ with CSPG antibody diluted 1:500 in $0.1 \mathrm{~mol} / \mathrm{l}$ phosphate buffer, $\mathrm{pH} 7.3 \mathrm{con}$ taining $1 \%$ BSA and $0.1 \%$ Tween 20 . Sections were washed in two changes of phosphate/BSA buffer, then incubated in neat normal goat serum for $15 \mathrm{~min}$ to reduce non-specific staining, prior to incubation for $2 \mathrm{~h}$ at $20^{\circ} \mathrm{C}$ with goat anti-rab- bit IgG coupled to $20 \mathrm{~nm}$ colloidal gold, diluted 1:20 in phosphate/BSA buffer containing $5 \%$ normal goat serum.

Specificity of tissue immunolabelling was confirmed by omission of the enzyme digestion step; preincubation of CSPG antibody with chondroitinase AC-digested chondroitin sulphate $\mathrm{A}$; omission or substitution of the primary antibody.

Collagen $I V$. The distribution of type IV collagen in undigested sections was demonstrated using rabbit anti-type IV collagen antiserum purchased from the Pasteur Institute, Lyon, France, using an indirect immunogold technique similar to that used to demonstrate CSPG. Antibody specificity was directed toward the globular domains of $\alpha 1$ (IV) and $\alpha 2(\mathrm{IV})$ collagen chains, triple helical and 7S domains of type IV collagen. Antiserum was diluted 1:500 for use.

Specificity of immunolabelling was confirmed by omission or substitution of the primary antibody.

After cationic gold or immunogold staining, sections were briefly washed in distilled water and counterstained in aqueous uranyl acetate for $3 \mathrm{~min}$ at $20^{\circ} \mathrm{C}$.

Image analysis. Single glomeruli from each case, sectioned through their maximal diameter were randomly selected for study. Sections were examined and photographed at $60 \mathrm{kV}$ using a Jeol $100 \mathrm{CX}$ transmission electron microscope (London, UK) as described previously [16]. Peripheral segments of nonoccluded capillary loops in sections cut perpendicular to the GBM in which epithelial slit-pores were visible were randomly selected for study. Quantitative analysis was performed on electron micrographs enlarged to final magnification 
of $\times 40,000-50,000$ using a MiniMagiscan image analysis system (Joyce-Loebl, Gateshead, UK). Analysis of staining from each case was based on measurements made along 1- $\mu \mathrm{m}$ lengths of GBM showing minor curvature, taken from at least six electron micrographs, representing at least $20 \mu \mathrm{m}$ length of glomerular capillary.

Membrane thickness was measured at $90^{\circ}$ to the plane of the GBM, at approximately $1-\mu \mathrm{m}$ intervals along the same $20 \mu \mathrm{m}$ length of GBM analysed for staining. Measurements were taken from the basal aspect of the foot processes to the inner margin of discernible basement membrane. Measurements did not include interposed GBM-like material in the subendothelium.

\section{Statistical analysis}

Membrane thickness (arithmetic mean) and staining density: gold particles/area $\left(\mu \mathrm{m}^{2}\right)$; gold particles/length $(\mu \mathrm{m})$ were compared in diabetic and "normal" GBM using unpaired $t$-test statistical analysis. Statistical significance was taken at or below the $5 \%$ level. The relationship between charge staining density and degree of proteinuria in individual patients was examined using Spearman rank correlation analysis. Data are given as mean $\pm 1 S D$.

\section{Results}

Morphology. The spectrum of glomerular pathological appearances in diabetic patients ranged from minor diffuse changes to severe nodular sclerosis as shown in Table 1 . Two patients receiving renal grafts showed evidence of recurrence of diabetic nephropathy in the transplanted kidneys. In patient 12 (19 weeks post-transplant) the changes were subtle, seen as characteristic thickening of only some glomerular capillary loops. By comparison, patient 13 at 5 years post-transplantation, showed diffuse GBM thickening and expansion of mesangial areas characteristic of diabetic nephropathy.

Charge staining. Despite the different glomerular morphologies seen in diabetic nephropathy, patterns of CG-stained anionic sites at both pH 2.5 and 5.8 showed consistent abnormalities compared with those typically seen in "normal" GBM (Fig. 1). Abnormal CG-staining patterns similar to those seen in diabetic nephropathy but not seen in other renal grafts (unpublished observations) were noted in both renal transplant patients. However, in patient 12 (19 weeks post-transplantation) the CG staining patterns were abnormal only in those glomerular capillary loops which showed thickening of the GBM. In other loops the distribution of anionic sites was similar to that seen in "normal" GBM. Due to these inconsistencies, patient 12 was excluded from GBM thickness measurements and CG staining analyses (see below).

At $\mathrm{pH} 2.5$, the linear array of regular GBM externa anionic sites (geometric mean interspace dis-
N.P.Goode et al.: Charge and structure in diabetic nephropathy

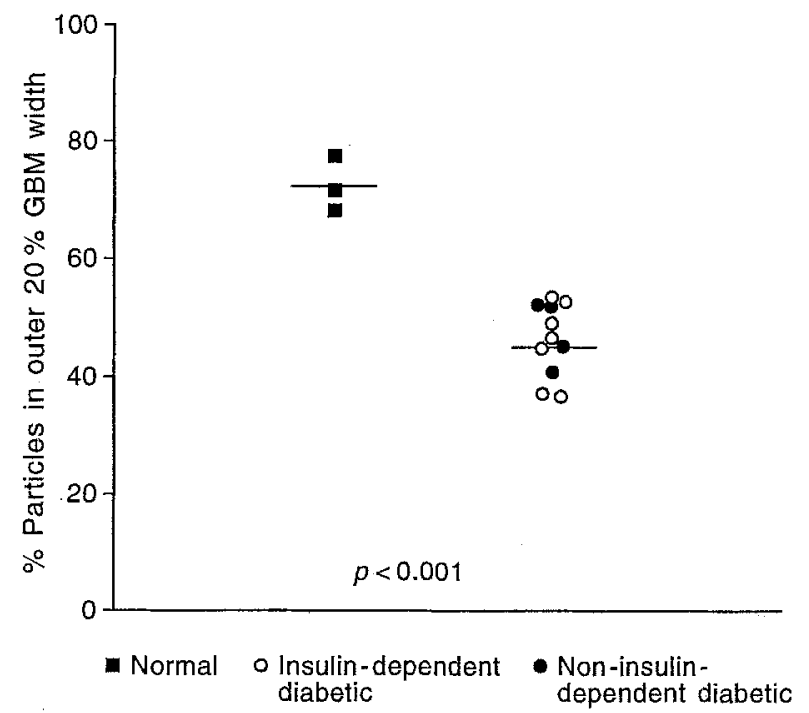

Fig. 2. Distribution of CG-stained anionic sites expressed at pH 2.5 in "normal" and diabetic GBM. Bar = group mean values. Points $=$ mean values in individual patients

tance: $67.8 \mathrm{~nm}$ ), typically seen in "normal" GBM (Fig. 1A), was replaced in all diabetic patients by randomly distributed anionic sites extending into the deeper layers of the thickened GBM (Fig.1B). The altered $\mathrm{pH} 2.5$ anionic site distribution is shown in Figure 2 as a shift in the percentage of particles present in the outer $20 \%$ of the GBM width. By comparison to "normal" GBM (mean percentage \pm SD: $72.4 \pm 4.4$ ), the GBM of diabetic patients showed a shift in the charge pattern distribution with a significant reduction in the percentage of sites located in the outer GBM $(46.4 \pm 6.1)(p<0.001)$. This pattern of staining was similar in insulin-dependent and noninsulin-dependent diabetes. In more thickened membranes the pattern of staining was due to increased charge expression, whilst in less expanded membranes the number of charged sites was similar to that seen in "normal" GBM.

At $\mathrm{pH}$ 5.8, anionic sites in patients with minor proteinuria and morphologically normal GBM were localized predominantly in the internal and external aspects of the GBM (Fig. 3A). By contrast, the external aspect of the thickened GBM in diabetic patients was heavily stained, with markedly reduced staining of the GBM interna (Fig. 3B). In some capillary loops this CG staining pattern was complicated by a highly charged layer of basement membrane-like material in the subendothelial region (Fig. 3B). This layer, which further contributed to thickening of the glomerular capillary wall, was continuous with mesangial matrix. Measurements of GBM thickness did not include this interposed matrix. A comparison of GBM thickness in control subjects $(283 \pm 23 \mathrm{~nm})$ and diabetic patients, $(484 \pm 173 \mathrm{~nm})(p<0.001)$ is shown in Figure 4 . The number of charged sites per unit area (charge density) was reduced only in those diabetic 


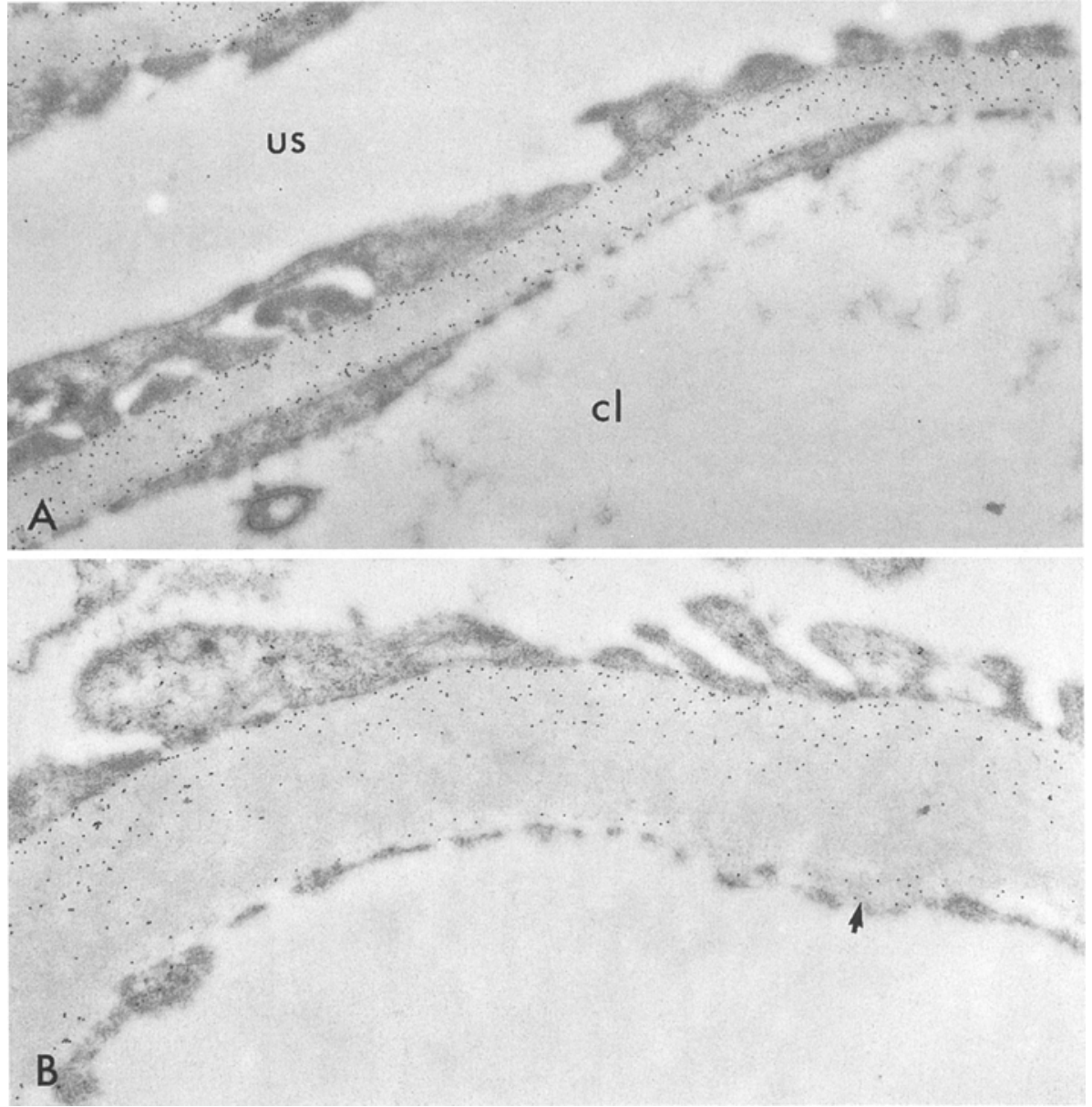

Fig.3A, B. Anionic sites demonstrated by $\mathrm{CG}$ at $\mathrm{pH}$ 5.8. A Sites are localised predominantly along the internal and external aspects of "normal" GBM. B The external aspect of thickened diabetic GBM is more heavily stained than "normal", whilst the GBM interna shows markedly reduced staining, patient 2. Arrow indicates charged basement membrane-like material in the subendothelium. (Both 34, $250 \times$ ). cl, Capillary lumen; us, urinary space patients with the greatest thickening of the GBM $(p>0.05)$. Charge per unit length was similar or increased compared with "normal" subjects (Fig. 4) $(p<0.05)$. The reduced GBM charge density in diabetic nephropathy being due to an increase of nonanionic GBM-like material in the inner aspect of the membrane, rather than loss of anionic sites (Fig. 3B). No correlation was observed between charge density and proteinuria $(p=0.873)$ or charge per unit length and proteinuria $(p=0.748)$.

Enzyme studies. Incubation of tissue sections in enzyme-containing buffers, with or without protease inhibitors, prior to $\mathrm{CG}$ staining at either $\mathrm{pH} 2.5$ or 5.8 , resulted in similar charge patterns.

The distribution of CG-stained anionic sites expressed at $\mathrm{pH} 2.5$ in "normal" and diabetic GBM, shown in Figure 1, is illustrated graphically in Figure 5. Heparitinase I pretreatment had no effect on anionic site expression in "normal" GBM at $\mathrm{pH} 2.5$. By contrast, anionic sites which were localized in the deeper layers of diabetic GBM at $\mathrm{pH} 2.5$, were heparitinase-sensitive (Fig.5). Removal of these deeper sites by heparitinase, which left externa sites intact, resulted in a charge distribution in diabetic GBM which resembled a "normal" GBM pH 2.5 pattern
(Fig.6). Heparitinase I pretreatment removed more than $90 \%$ of all anionic sites expressed at pH 5.8 in "normal" and diabetic GBM.

Pretreatment of tissue with chondroitinase $\mathrm{AC}$ did not significantly reduce the number of $\mathrm{CG}$ stained sites in diabetic or "normal" GBM at either $\mathrm{pH} 2.5$ or 5.8. Anionic sites expressed at $\mathrm{pH} 5.8$ in association with interstitial collagen fibres and platelet granules were removed by pretreatment with this enzyme. Evidence of chondroitinase $\mathrm{AC}$ activity was further demonstrated by the requirement of this enzyme to unmask antigenic determinants on CSPG constituents of the GBM, platelet granules and interstitial collagen fibres, prior to immunostaining with a commercially prepared antibody which had been raised against chondroitinase-digested CSPG as immunogen.

\section{Immunoelectron microscopy}

Chondroitin sulphate proteoglycans. CSPG components were demonstrated by immunogold staining in mesangial matrix and localised mainly along the external aspect of "normal" GBM (Fig.7A). The pattern of GBM staining was similar in all three control 


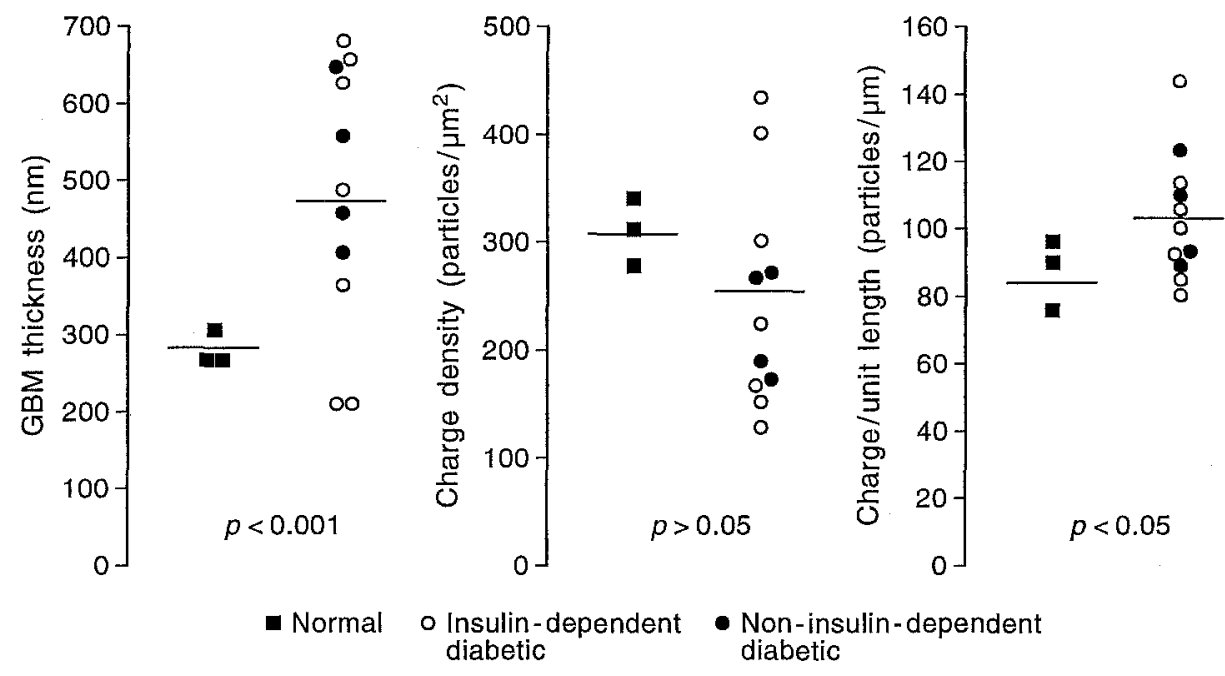

Fig. 4. Membrane thickness, charge density and charge per unit length in "normal" compared with diabetic GBM. Bar = group mean values. Points $=$ mean values in individual patients

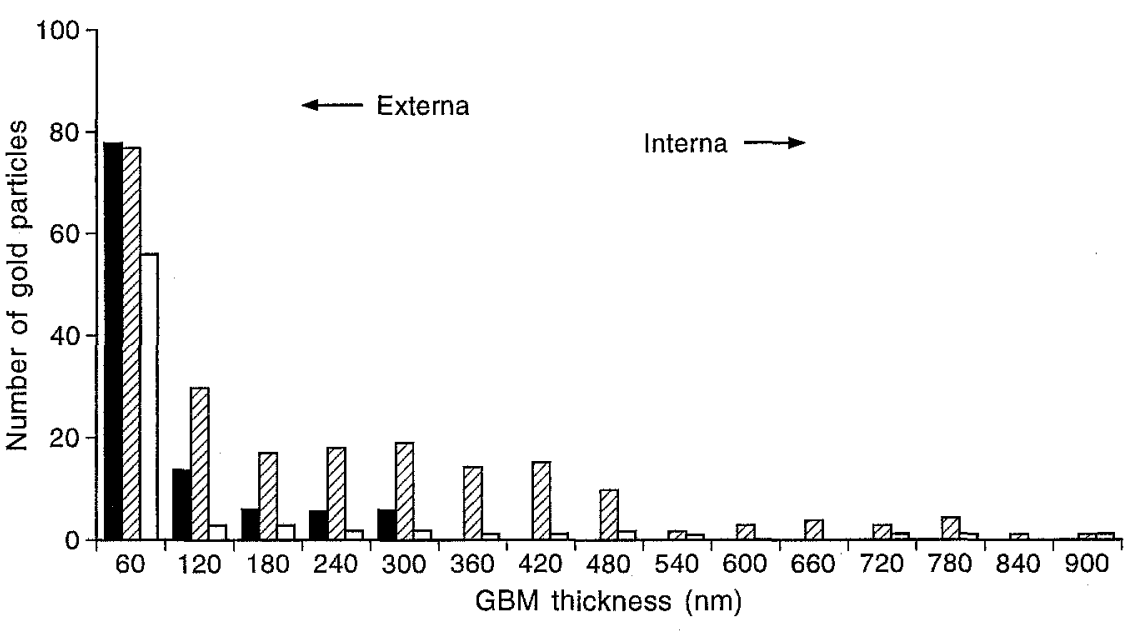

Normal
Fig. 5. Graphical representation of the CGstained anionic site distribution expressed at pH 2.5 in "normal" (Fig. 1A), diabetic GBM (Fig. 1B) and in heparitinase I pretreated diabetic GBM as shown in Figure 6. biopsies showing "normal" GBM morphology. Although CSPG sites were scarce in some GBM segments, most segments showed a uniform staining distribution. Quantitative analysis was performed on one representative case (patient 14). Measurements were made in those GBM segments which showed more uniform staining (Table 2).

In those diabetic patients who showed the greatest thickening of GBM $(>400 \mathrm{~nm})$, the CSPG staining pattern showed consistent abnormalities. Quantitative analysis was performed on one representative case (patient 9). As shown in Table 2, the CSPG staining pattern showed an increase in the absolute number (particles/ $\mu \mathrm{m}$ length GBM) of immunogoldstained sites compared with "normal", although the staining density (particles $/ \mu \mathrm{m}^{2}$ ) was actually reduced. The distribution of CSPG was more random than that seen in "normal" GBM, and extended deeper into the thickened membrane (Table 2, Fig. 7B). In diabetic GBM which showed only a moderate increase in thickness $(<400 \mathrm{~nm})$ a disrupted CSPG pattern was not apparent.
Staining was eliminated by omission, substitution, or neutralization of the primary antibody, and by omission of the enzyme digestion step.

Collagen IV. A different distribution of type IV collagen was also observed in "normal" and diabetic tissue (Table 2, Fig. 8). As with CSPG, the GBM staining pattern was similar in all three control biopsies showing "normal" GBM morphology. Quantitative analysis was performed on one representative case (patient 14). In contrast to CSPG, localized along the GBM externa, collagen IV staining was restricted to the inner aspect of "normal" GBM (Table 2, Fig. 8A).

In a manner similar to CSPG, an abnormal collagen IV staining pattern was manifested in those GBM segments which showed greatest thickening $(>400 \mathrm{~nm})$. Quantitative analysis was performed on one representative case (patient 9). As with CSPG, the absolute number of immunogold-stained collagen IV sites per micron length of GBM was increased, but the overall density of staining (particles/ $\mu \mathrm{m}^{2}$ ) was reduced. The distribution of staining was 


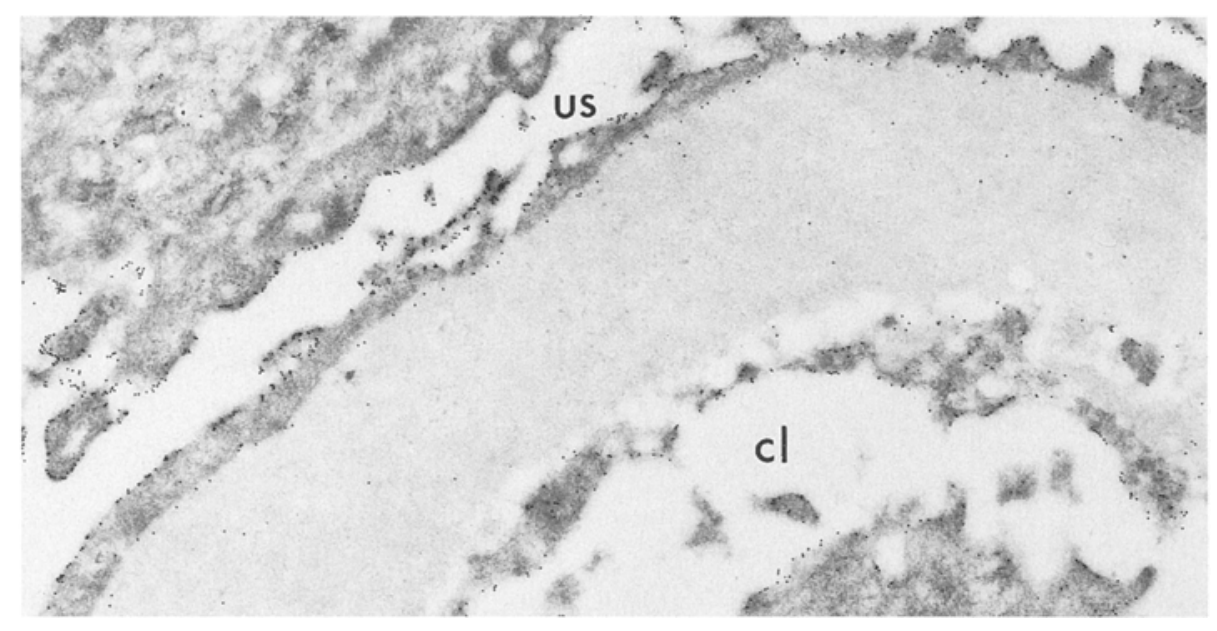

Fig. 6. Heparitinase treatment prior to $\mathrm{CG}$-staining at $\mathrm{pH} 2.5$. The majority of anionic sites in the deeper layers of the membrane are removed, but externa sites remain intact. Compare with same segment in serial section (Fig. 1B), patient $4(31,500 \times)$. cl, Capillary lumen; us, urinary space
Table 2. Distribution, absolute number and labelling density of CSPG and collagen IV immunogold staining in representative "normal" and diabetic GBM

\begin{tabular}{|c|c|c|c|c|}
\hline \multirow{2}{*}{$\begin{array}{l}\% \text { GBM } \\
\text { thickness }\end{array}$} & \multicolumn{2}{|c|}{ CSPG distribution $^{\mathrm{a}}$} & \multicolumn{2}{|c|}{ Collagen IV distribution ${ }^{\mathrm{a}}$} \\
\hline & "normal"b & diabetic $^{c}$ & "normal"b & diabetic $^{c}$ \\
\hline interna-20 & $3.6 \pm 2.3$ & $2.2 \pm 0.8$ & $69.4 \pm 5.7$ & $22.8 \pm 2.9$ \\
\hline $21-40$ & $4.6 \pm 1.7$ & $8.4 \pm 2.6$ & $12.4 \pm 2.2$ & $22.6 \pm 2.1$ \\
\hline $41-60$ & $6.8 \pm 1.5$ & $23.0 \pm 3.1$ & $7.6 \pm 2.0$ & $17.2 \pm 5.4$ \\
\hline $61-80$ & $17.8 \pm 4.6$ & $29.0 \pm 4.9$ & $7.0 \pm 2.9$ & $19.8 \pm 2.7$ \\
\hline 81-externa & $67.2 \pm 6.8$ & $39.4 \pm 3.4$ & $3.6 \pm 1.7$ & $17.6 \pm 2.5$ \\
\hline $\begin{array}{l}\text { No. particles/ } \\
\mu \mathrm{m}\end{array}$ & $14.4 \pm 2.3$ & $27.7 \pm 3.8$ & $24.0 \pm 3.2$ & $39.7 \pm 4.1$ \\
\hline $\begin{array}{l}\text { No, particles/ } \\
\mu^{2}\end{array}$ & $72.0 \pm 5.6$ & $55.3 \pm 8.6$ & $120.0 \pm 8.7$ & $99.0 \pm 6.7$ \\
\hline
\end{tabular}

Measurements based on $20 \mu \mathrm{m}$ length GBM in each case. Data are mean $\pm 1 \mathrm{SD}$.

a Percentage of particles; ${ }^{b}$ Patient 14 ; ${ }^{c}$ Patient 9

more random in diabetic compared with "normal" GBM, extending across the entire width of the thickened membrane (Table 2, Fig. 8B).

The density of staining of collagen IV in mesangial matrix of diabetic glomeruli was increased compared with "normal" except in sclerotic areas which were unstained.

Staining was eliminated by omission or substitution of the primary antibody.

\section{Discussion}

The CG technique has been shown to be a reliable method for the ultrastructural localization and quantitation of glomerular anionic components in postembedded renal biopsy tissue [16]. In contrast to studies using other markers, CG applied under different conditions of $\mathrm{pH}$ and electrolyte concentration results in distinct patterns of staining, due to charge expression by tissue components with different physicochemical characteristics. Our previous studies have demonstrated consistent patterns of glomerular an- ionic charge distribution at $\mathrm{pH} 2.5$ and 5.8 in patients with only minor proteinuria and normal GBM morphology $[16,25]$. In the absence of normal biopsy tissue these typical patterns are regarded as "normal". Anionic site distribution may be altered in glomerular disease due to pathological abnormalities arising from structural rearrangement of GBM constituents or deposition of extrinsic material. In diabetic nephropathy the alterations in GBM charge patterns are unique. The demonstration of anionic sites extending deep into the thickened diabetic GBM at $\mathrm{pH} 2.5$, together with loss of GBM interna staining at $\mathrm{pH} 5.8$, seen in all the diabetic cases examined, irrespective of the degree of proteinuria, has not been seen in any other glomerulopathy in our study of over 200 random renal biopsies encompassing a broad spectrum of disease [26].

The presence of additional heparitinase-sensitive anionic sites and CSPG constituents extending from the external aspect into the deeper layers of the membrane provides evidence of restructuring and thickening of the epithelial side of the diabetic membrane. Loss of interna staining from the diabetic GBM at $\mathrm{pH} 5.8$ is not due to charge site loss, but instead, appears to be due to displacement of anionic components by incorporation of non-anionic GBM constituents on the endothelial side of the membrane. This observation contrasts with the decreased number of stained sites in both the lamina rara externa (LRE) and lamina rara interna (LRI) of diabetic GBM, reported by investigators using cuprolinic blue or polyethyleneimine [27] as cationic markers. This discrepancy may be explained by differences in analysis of staining patterns using a definitive marker such as $\mathrm{CG}$, and markers producing a variable signal which is open to subjective interpretation. Vernier et al. [12], in their study using cuprolinic blue, did not quantitate smaller, less densely stained sites which were noted to be increased in the lamina densa of diabetic subjects. By contrast, all CG stained sites were quantitated in the present study, including 


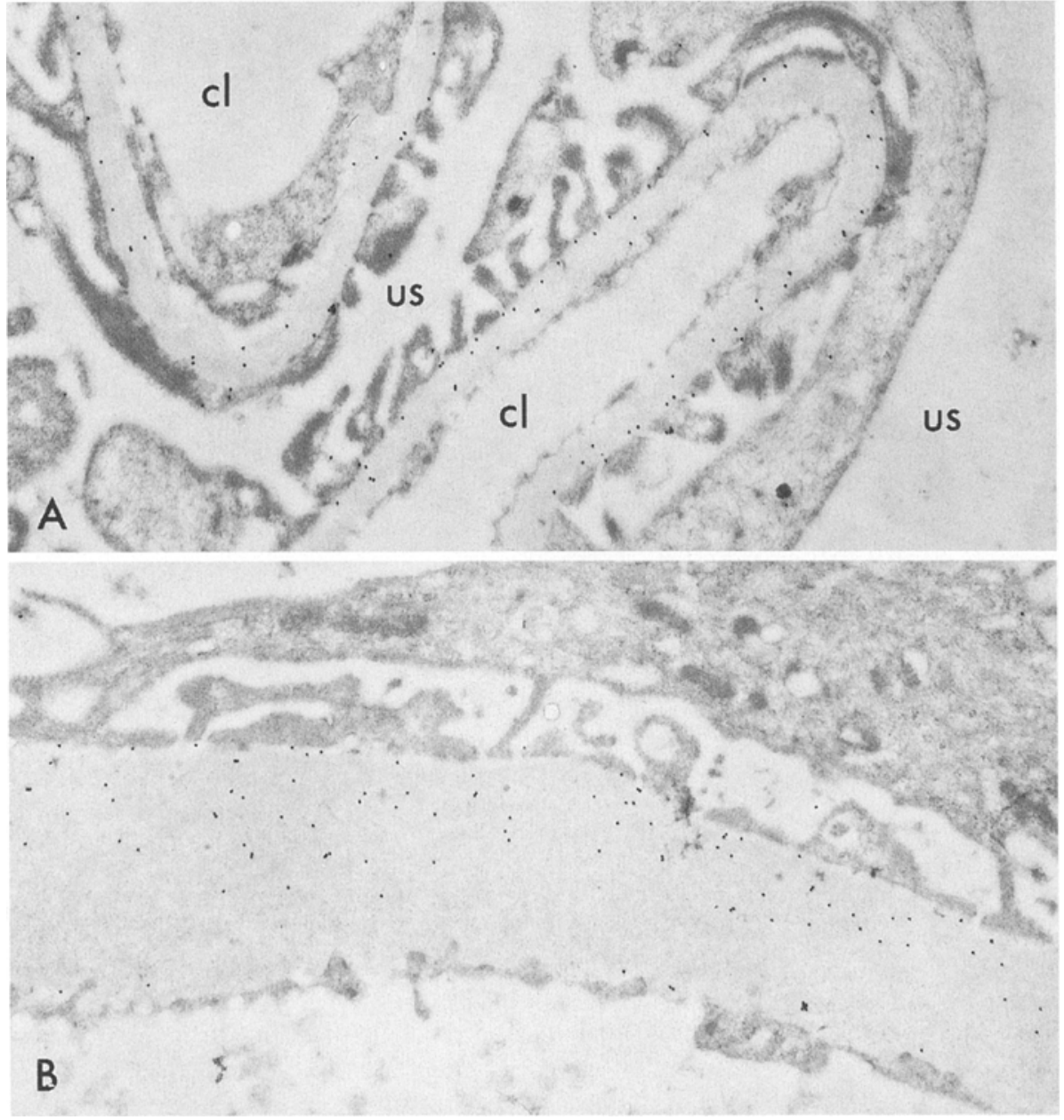

Fig. 7. A CSPG components are localised mainly along the external aspect of "normal" GBM. B In diabetic GBM, increased staining extends deep into the thickened membrane, patient 9 (Both $34,500 \times$ ). cl, Capillary lumen; us, urinary space those which extended into the deeper layers of the thickened diabetic GBM.

We have not distinguished lamina densa and laminae rarae, due to a lack of clear demarcation of these regions in lightly counterstained tissue. A highly charged subendothelial layer of CG-stained basement-membrane-like material, continuous with mesangial matrix was a prominent feature within the non-occluded loops of diabetic glomeruli. Makino et al. [5] described a similar feature, revealed by high resolution scanning microscopy of glomeruli from patients with diabetic nephropathy, and also noted in streptozotocin-induced diabetic rats. Although electron micrographs published by Vernier et al. [12], showed a clearly discernible subendothelial layer, stained with cuprolinic blue, this feature was not commented upon, apparently being included as an integral part of the LRI in their study.

A reduced glomerular HSPG content, demonstrated biochemically in isolated glomeruli from autopsy kidneys of patients with advanced diabetic nephropathy, compared with control subjects [28] may correspond with our observations of reduced CG staining of diabetic glomeruli in areas of mesangial expansion and nodular sclerosis.
Vernier et al. [12] examined the effects of enzyme pretreatment on GBM anionic sites in post-fixed, pre-embedded blocks of normal neonatal tissue, but not diabetic GBM. We have applied enzymes to post-fixed, post-embedded ultrathin sections of both diabetic and "normal" tissue. The major sulphated GBM anionic constituent stained by either CG or cuprolinic blue under critical electrolyte concentration conditions [22], appears to be HSPG since heparitinase pretreatment removes more than $90 \%$ of stainable sites. Although HSPG supposedly constitutes a glomerular filtration charge barrier, disturbance of this barrier, seemingly, does not relate to the degree of proteinuria in diabetic nephropathy.

The sensitivity of a sub-population of anionic sites expressed at $\mathrm{pH} 2.5$ in diabetic GBM to heparitinase pretreatment suggests that these sites are expressed by newly formed component complexes which are stabilised by HSPG.

The presence of CSPG in GBM is controversial, having been considered by some investigators [1719] to be a component which is absent in normal mature GBM (see below). The influence of chondroitinase AC on GBM anionic site expression was equivocal due to difficulties of precise quantitative compari- 


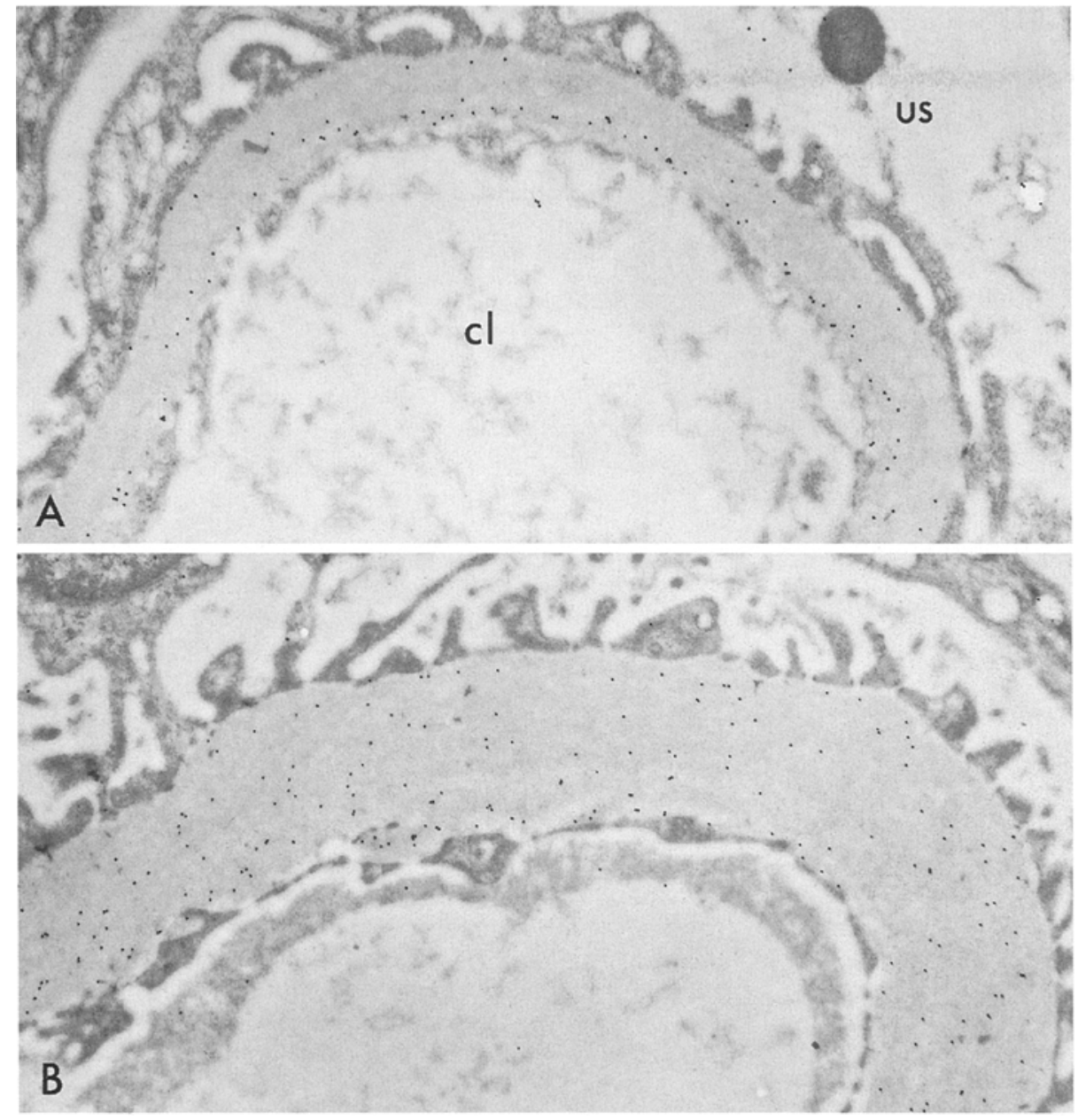

Fig. 8. A Type IV collagen is restricted to the inner aspect of "normal" GBM. B In diabetic GBM, increased staining extends across the entire width of the thickened membrane, patient 9 (Both $34,500 \times$ ) cl, Capillary lumen; us, urinary space son of anionic sites in serial sections in which enzymatic removal of anionic sites was subtle. Persistence of charged sites after chondroitinase AC digestion may be due to the presence of CSPG components which are complexed with other highly charged proteoglycans such as HSPG [23]. Alternatively, the GAG-core protein stub remaining after enzyme digestion may contain sufficient residual charge groups to retain binding of the cationic gold marker. Activity of the chondroitinase enzyme was confirmed by removal of CG-stained sites associated with interstitial collagen fibres and platelet granules which are known to contain diverse CSPG constituents. Immunogold staining, which paradoxically required pretreatment of tissue with chondroitinase AC to expose antigenic sites, provided a positive localization of CSPG in the external aspect of control GBM and extending into the deeper layers of diabetic GBM. We have not been able to demonstrate CSPG in normal adult rat GBM, suggesting probable species differences in the distribution of this antigen (unpublished data). Studies by McCarthy and Couchman [17] in rats had previously concluded that CSPG is not a component of normal mature GBM. Interestingly however, these authors describe the presence of CSPG in the GBM of streptozotocin-induced diabetic rats [18]. Vernier et al. [12] reported a $26 \%$ reduction of stainable LRI sites, but no effect on LRE sites following chondroitinase ABC pretreatment of normal infant kidney, concluding that CSPG may be a minor anionic constituent in the LRI of human GBM. This localization of CSPG in the GBM interna contrasts with our demonstration of CSPG restricted to the GBM externa. The discrepancy may be due to maturational differences in GBM structure, similar to those in prenatal and postnatal rats described by McCarthy et al. [19].

The demonstration of increased type IV collagen staining in the thickened GBM and expanded mesangial matrix of non-sclerosed areas of diabetic glomeruli in the present study, supports observations by other investigators [5, 29-31]. An increased glomerular synthesis and content of type IV collagen has been shown biochemically in diabetes $[29,30]$. Increased immunocytochemical staining for GBM type IV collagen has also been shown by light [31] and immunoelectron [5] microscopy. Investigations of type IV collagen distribution in diabetic GBM have, however, produced diverse results attributable to differences in the species studied and the antibodies used $[5,32]$. 
Employing an antibody directed against the globular domains of $\alpha 1$ (IV) and $\alpha 2$ (IV) collagen chains, and triple helical and 7S domains of type IV collagen, we have demonstrated that collagen IV staining is limited predominantly to the internal aspect of "normal" GBM, whilst in diabetic GBM, staining is more randomly distributed across the entire membrane thickness. By contrast, Makino et al. [5] demonstrated immunogold staining for type IV collagen, heaviest in the lamina densa but extending across the entire width of control GBM, which was unchanged in patients with diabetic nephropathy. Bendayan et al. [33] demonstrated that collagen IV staining was restricted to the central lamina densa of normoglycaemic rats, but was preferentially localized along the internal aspect of the thickened GBM of streptozotocin-induced diabetic rats. The antiserum used in that study [33] was directed against an antigen extracted from a mouse EHS tumour which reputedly contains only $\alpha 1$ (IV) and $\alpha 2$ (IV) chains [34]. In all these studies, non-reactive portions of the GBM represent site-specific arrays of the collagenous meshwork, composed of $\alpha$-chains which do not react with the antiserum used [35].

Inconsistencies in the localization of CSPG and collagen IV present problems of comparison between different studies. However, in this study, detection of these components, restricted to the externa or interna, respectively of "normal" GBM, makes them ideal markers for the demonstration of membrane component rearrangement which is associated with thickening of the diabetic GBM. Redistribution of these markers and CG-stained anionic sites in diabetic GBM, suggests that thickening results from changes derived from both the internal and external aspects of the GBM. Thus, CSPG and externa anionic sites expressed at $\mathrm{pH} 2.5$ are relocated inwards in the diabetic GBM, whilst collagen IV and interna anionic sites expressed at $\mathrm{pH} 5.8$ are displaced outwards by uncharged basement-membrane-like material. In diabetic nephropathy, the GBM interna, by virtue of this lack of charge, superficially resembles areas of sclerosed mesangial matrix. However, unlike GBM interna, sclerosed areas do not stain at all for collagen IV.

Alterations of the CG-staining patterns are seen in the absence of accompanying CSPG and collagen IV redistribution in glomerular capillaries with minimally thickened GBM. The presence of characteristic CG-staining patterns in diabetic nephropathy cases with minor or heavy proteinuria, mild or severe glomerular pathology, and including patients with renal grafts in whom there was recurrence of disease, suggests that the $C G$ staining technique may provide an early marker of glomerular renal disease in diabetic patients.

The CG staining technique may be of value in the assessment of GBM structural alterations in renal bi- opsies from patients presenting with early clinical manifestations of renal disease such as microalbuminuria, and from patients receiving dual pancreatic and renal grafts.

Acknowledgements. This work was supported by the Yorkshire Kidney Research Fund.

\section{References}

1. Osterby R, Gundersen HJG, Nyberg G, Aurell AM (1987) Advanced diabetic nephropathy: quantitative structural characterization of non-occluded glomeruli. Diabetes 36 : 612-619

2. Spiro RG (1988) Pathogenesis of diabetic glomerulopathy: a biochemical view. In: Mogensen CE (ed) The kidney and hypertension in diabetes mellitus. Nijhoff, Boston, pp 117130

3. Karttunen T, Risteli J, Autio-Harmainen H, Ristali L (1986) Effect of age and diabetes on type IV collagen in human kidney cortex. Kidney Int 30: 586-591

4. Kim Y, Kleppel MM, Butkowski R, Mauer SM, Weislander J, Michael AF (1991) Differential expression of basement membrane collagen chains in diabetic nephropathy. Am J Pathol 138: 413-420

5. Makino H, Yamasaki Y, Haramoto T et al. (1993) Ultrastructural changes of extracellular matrices in diabetic nephropathy revealed by high resolution scanning and immunoelectron microscopy. Lab Invest 68: 45-55

6. Nicholls K, Mandel TE (1989) Advanced glycosylation end-products in experimental murine diabetic nephropathy: effect of islet isografting and of aminoguanidine. Lab Invest 60: $486 \sim 489$

7. Kanwar YS, Rosenzweig LJ, Jakubowski ML (1983) Distribution of de novo synthesized sulfated glycosaminoglycans in the glomerular basement membrane and mesangial matrix. Lab Invest 49: 216-225

8. Kanwar YS, Rosenzweig LJ, Linker A, Jakubowski ML (1983) Decreased de novo synthesis of glomerular proteoglycans in diabetes. Biochemical and autoradiographic evidence. Proc Natl Acad Sci USA 80: 2272-2277

9. Wahl P, Deppermann D, Hasslacher C (1982) Biochemistry of the glomerular basement membrane of normal and diabetic human. Kidney Int 21: 744-749

10. Shimomura H, Spiro RG (1987) Studies on macromolecular components of human glomerular basement membrane and alterations in diabetes: decreased levels of heparan sulphate proteoglycan and laminin. Diabetes 36: 374-381

11. Parthasarathy N, Spiro RG (1982) Effect of diabetes on the glycosaminoglycan component of the human glomerular basement membrane. Diabetes 31: 738-741

12. Vernier RL, Steffes MW, Sisson-Ross S, Mauer SM (1992) Heparan sulphate proteoglycan in the glomerular basement membrane in type I diabetes mellitus. Kidney Int 41: 1070-1080

13. Deckert TD, Feldt-Rasmussen B, Djurup R, Deckert M (1988) Glomerular size and charge selectivity in insulin-dependent diabetes mellitus. Kidney Int 33: 100-106

14. Abrahamson DR (1986) Recent studies on the structure and pathology of basement membranes. J Pathol 149: 257A (Abstract)

15. Weber M (1992) Basement membrane proteins. Kidney Int 41: $620-628$

16. Goode NP, Shires M, Aparicio SR, Davison AM (1991) Cationic colloidal gold: a novel marker for the demonstra- 
tion of glomerular polyanion status in routine renal biopsies. Nephrol Dial Transplant 6: 923-930

17. McCarthy KJ, Couchman JR (1990) Basement membrane chondroitin sulfate proteoglycans: localization in adult rat tissues. J Histochem Cytochem 38: 1479-1486

18. McCarthy KJ, Abrahamson DR, Couchman JR (1991) Basement membrane-specific chondroitin sulfate proteoglycan is abnormally associated with the glomerular capillary basement membrane in streptozotocin-induced diabetic rats. Diabetes 40: 129A (Abstract)

19. McCarthy KJ, Bynum K, St. John PL, Abrahamson DR, Couchman JR (1993) Basement membrane proteoglycans in glomerular morphogenesis: chondroitin sulphate proteoglycan is temporally and spatially restricted during development. J Histochem Cytochem 41: 401-414

20. Dyson EH, Will EJ, Davison AM, O'Malley AH, Shepherd HT, Jones RG (1992) Use of the urinary protein creatinine index to assess proteinuria in renal transplant patients. Nephrol Dial Transplant 7: 450-452

21. Goode NP, Shires M, Davison AM (1992) Preparation and use of the poly-L-lysine gold probe: a differential marker of glomerular anionic sites. Histochemistry 98: 67-72

22. Scott JE, Dorling J (1965) Differential staining of acid glycosaminoglycans (mucopolysaccharides) by Alcian blue in salt solutions. Histochemie 5: 221-233

23. Ruoshlahti E (1988) Structure and biology of proteoglycans. Ann Rev Cell Biol 4: 229-295

24. Bertolotto A, Palmucci L, Gagliano A, Mogini T, Tarone G (1986) Immunofluorescence localization of chondroitin sulphate in normal and pathological muscle. J Neurol Sci 73: 233-244

25. Danscher G, Nörgaard JOR (1983) Light microscopic visualisation of colloidal gold on resin-embedded tissue. J Histochem Cytochem 31: 1394-1398

26. Goode NP, Shires M, Aparicio SR, Davison AM (1994) GBM derangement in diabetic nephropathy is reflected by characteristic charge patterns. Nephrology Dial Transplant 9: 850 (Abstract)
27. Rohrbach R (1986) Reduced content and abnormal distribution of anionic sites (acid proteoglycans) in the diabetic glomerular basement membrane. Virchows Arch B-Cell Pathol 51: 127-135

28. Shimomura H, Spiro RG (1987) Studies on macromolecular components of human glomerular basement membrane and alterations in diabetes. Diabetes 36: 374-381

29. Ihm CG, Lee GSL, Nast CC et al. (1992) Early increased renal procollagen alpha-1 (type IV) mRNA levels in streptozotocin induced diabetes. Kidney Int 41: 768-777

30. Karttunen T, Risteli J, Autio-Hermainen H, Ristali L (1986) Effect of age and diabetes on type IV collagen and laminin in human kidney cortex. Kidney Int 30: 586-591

31. Falk RJ, Scheinman JI, Mauer SM, Michael AF (1983) Polyantigenic expansion of the basement membrane constituents of diabetic nephropathy. Diabetes 32[Suppl 2]:34-39

32. Desjardins M, Gros F, Weislander J, Gubler M-C, Bendayan M (1990) Heterogeneous distribution of monomeric elements from the globular domain (NC1) of type IV collagen in renal basement membranes as revealed by high resolution quantitative immunocytochemistry. Lab Invest 63: 637-646

33. Bendayan M (1985) Alteration in the distribution of type IV collagen in glomerular basal laminae in diabetic rats as revealed by immunocytochemistry and morphometrical approach. Diabetologia 28: 373-378

34. Kleppel MM, Michael AF, Fish AJ (1986) Comparison of non-collagenous type IV collagen components in the human glomerulus and EHS tumor. Biochim Biophys Acta 883: 178-189

35. Zhu D, Youngki K, Steffes MW, Groppoli TJ, Butkowski RJ, Mauer SM (1994) Application of electron microscopic immunocytochemistry to the human kidney: distribution of type IV and type VI collagen in normal human kidney. $J$ Histochem Cytochem 42: 577-584 